\title{
Adaptive interferometer with optoelectronic feedback
}

Igor A. Sokolov, Mikhail A. Bryushinin

A.F. loffe Physical Technical Institute, Russian Academy of Sciences

194021 Polytekhnicheskaya, 26, St.-Petersburg, Russia

Detection of low-amplitude acoustic vibrations of real objects, such as ultrasonic transducers, composite materials, biological objects, metal production, MEMS is an important scientific and technical problem. Homodyne laser vibrometers are suitable for practical applications in this regard for their high sensitivity which is limited in principle only by shot-noise of the laser used [1]. These vibrometers allow detection at a distance, with high spatial localization of the measurement region and wide frequency range. However, their utilization is restrained by several problems such as slow phase drifts in the interferometer arms due to environmental reasons, necessity of fine optical adjustment and suppression of laser amplitude noise. Wide-gap photorefractive materials offer an elegant way to solve the problem of keeping operation interferometer point constant [2-4]. The crystal replaces the conventional beamsplitter and can be controlled not only electrically but also optically, i.e., based on the principles of nonlinear optics. In addition, it can be multilayered. Such a multilayered adaptive beamsplitter is nothing else but a volume dynamic hologram recorded by the reference and signal waves. Adaptive detectors based on the effect of the non-steady-state photo-electromotive force (photo-EMF) [5] are promising for sensor applications. The nonstationary holographic photocurrent (or non-steady-state photo-EMF) is a holography related effect and it appears in a semiconductor material illuminated by an oscillating light pattern. Such illumination is usually created by two coherent light beams one of which is phase modulated with frequency $\omega$.

Among the number of the nonlinear phenomena observed in photorefractive crystals there are effects which appearance or specific behavior is determined by additional feedback loop. Stabilization of a holographic setup was one of the earliest and the most important application of the optoelectronic feedback. It is implemented using the detection scheme and the piezoelectric mirror placed in one of the arms of the setup. The mirror is driven by the amplified voltage of the detector providing the necessary feedback loop. Such feedback keeps the difference of the optical path lengths to be constant and correspondingly stabilizes position of the interference fringes. As a result the holograms are recorded with high modulation index.

Generally speaking, the implementation of the feedback in optical schemes reveals features typical to those in common dynamical systems (mechanical, electronic, biological) considered in the theory of oscillation and control theory [6, 7]. For example, the negative feedback in Michelson interferometer provides the ability to linearize the sensor (photodiode) output allowing detection of large displacements [8]. The conventional electronic operational amplifier demonstrates similar behavior with the feedback loop. Various optical schemes with positive feedback such as laser, molecular cell inside Fabry-Perot resonator, hybrid schemes with birefringent materials [9] reveal the oscillatory or bistable behavior which also has analogs in electronics and mechanics (harmonic oscillators, triggers). Since the presence of the properly chosen feedback in dynamical system not only improves its performance but can also provide observation of new nonlinear phenomena, we have applied feedback operation concept to the sensor based on the effect of the non-steady-state photo-EMF.

The non-steady-state photoelectromotive force (photo-EMF) is a holography related effect and it appears in a semiconductor material illuminated by an oscillating light pattern. Such illumination is usually created by two coherent light beams one of which is phase modulated with frequency $\omega$. The alternating current is resulted from the periodic relative shifts of the photoconductivity and space charge gratings which arise in the crystal's volume under illumination. Like the holographic recording in photorefractive crystals this effect demonstrates adaptive properties that promote its application in such areas as vibration monitoring, velocimetry, etc. [10]. In contrast to the holographic methods this technique allows the direct transformation of phase modulated optical signals into the electrical current and can be applied for characterization of centrosymmetrical and even amorphous materials. Since the photocurrent is originated from the interaction of both the photoconductivity and space charge gratings a lot of photoelectric parameters can be measured [11]. 
In this study we introduce the positive feedback and organize the generation mode operation in the interferometer using the non-steady-state photo-EMF detector (Figure 1). In contrast to techniques developed in Refs. [12-18] we do not use any dither signals and synchronous detection. Our approach implies the utilization of a conventional wideband amplifier in the electronic circuit. Both the diffusion and drift regimes are investigated theoretically and experimentally in $\mathrm{Bi}_{12} \mathrm{SiO}_{20}$ crystal.
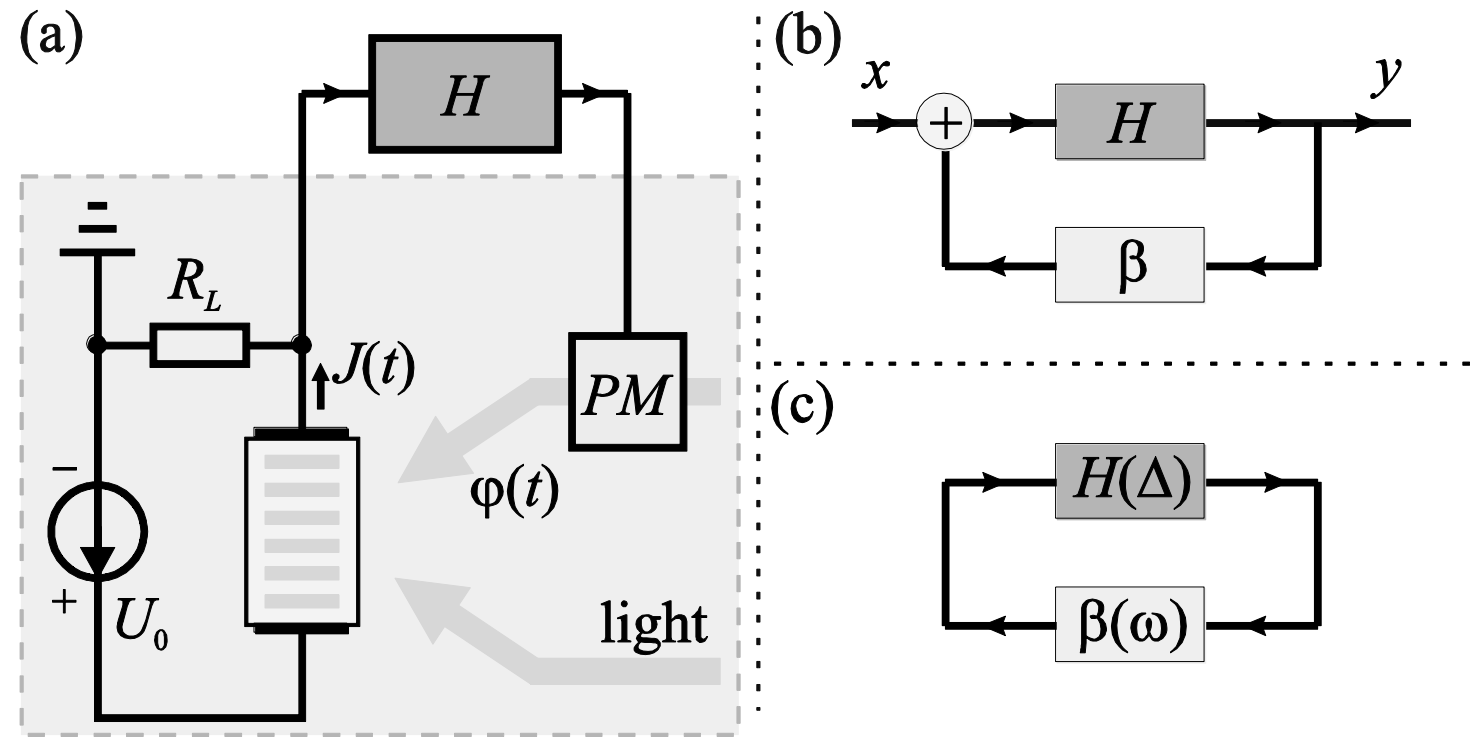

Figure 1. Block diagrams illustrating closed-loop operation of the interferometric scheme studied in this paper (a), general feedback structure (b), and general oscillator scheme (c). PM is the phase modulator.

Let us start the analysis of our interferometric scheme (Fig. 1, a) with formulation of the problem in terms of oscillation theory and control theory [6-8]. Dynamical systems are often represented by block diagrams, which have a unique algebra and set of transformations. The block diagrams are used because they are a shorthand pictorial representation of the cause-and-effect relationship between the input and output in a real system, and they are a convenient method for characterizing the functional relationships between components. One important arrangement is the general feedback form illustrated in Fig. 1, b. In this figure $\mathrm{H}$ and $\beta$ represent the transfer functions from the input to the output of the systems contained within their respective boxes. The overall transfer function is as follows:

$$
\mathrm{T}=\mathrm{H} /(1-\mathrm{H} \beta)
$$

Product $\mathrm{H} \beta$ is often called loop gain. When this product tends to unity the transfer function diverges what corresponds to the appearance of an instability and unlimited growth of oscillations in the closed-loop system.

The basic form of a harmonic oscillator is an amplifier with the output attached to a narrow-band filter $\beta=\beta(\omega)$ ], and the output of the filter attached back to the input of the amplifier (Fig. 1, c). The unlimited growth of oscillations is usually restricted by nonlinearity of the amplifier observed at large amplitudes, i.e. $\mathrm{H}=\mathrm{H}(\Delta)$. Relation

$$
H(\Delta) \beta(\omega)=1
$$

is the main equation (Barkhausen equation) defining the amplitude $\Delta_{\mathrm{g}}$ and frequency $\omega_{\mathrm{g}}$ in the steady-state oscillation regime. So, our main goal in this section is to obtain the analogous equation for the interferometric scheme using the non-steady-state photo-EMF detector and feedback loop (Fig. 1, a) and calculate the corresponding oscillation amplitudes and frequencies.

Let us assume the crystal is illuminated by an oscillating interference pattern created by two light beams one of which is phase modulated with amplitude $\Delta$ and frequency $\omega$ :

$$
\mathrm{I}(\mathrm{x}, \mathrm{t})=\mathrm{I}_{0}(1+\mathrm{mcos}(\mathrm{Kx}+\Delta \cos \omega \mathrm{t}))
$$

Here $I_{0}$ is the average intensity, $m$ and $K$ are the contrast and spatial frequency of the interference pattern. Such illumination excites an ac current in the crystal's volume via non-steady-state photo-EMF effect. This current produces the corresponding voltage at load resistor, which is then amplified and transferred into phase modulation with amplitude $\Delta$ using the electrooptic modulator:

$$
\mathrm{D}=\mathrm{JR}_{\mathrm{L}} \mathrm{H} /\left(\mathrm{U}_{\pi} / \pi\right)
$$


where $U_{\pi}$ is the half-wave voltage of the phase modulator, $R_{L}$ is the load resistance, and $H$ is the gain factor of the amplifier. So the feedback loop is closed. We can easily rewrite Eq. (4) in the form analogous to Barkhausen equation (2):

$$
\mathrm{H}\left(\pi \mathrm{J}^{\omega}(\omega, \Delta) \mathrm{R}_{\mathrm{L}} / \mathrm{U}_{\pi} \Delta\right)
$$
range:

We shall consider the ideal amplifier with the flat frequency transfer function and infinite dynamic

$$
\mathrm{H}=\text { const }\left(\omega, \mathrm{U}_{\text {in }}\right)
$$

In this case the frequency and amplitude of oscillations is defined by the frequency response and nonlinearity of the effect of the non-steady-state photo-EMF but not by the amplifier.

They usually differ two basic mechanisms of the space charge formation -- diffusion and drift ones [19]. Let us proceed the analysis of the former.

For diffusion mechanism of recording no external voltage is applied to the sample and the amplitude of the non-steady-state photocurrent excited in the crystal with electron conductivity is given by the following expression:

$$
J^{\omega}=\frac{-S m^{2} J_{0}(\Delta) J_{1}(\Delta) \sigma_{0} E_{D} i \omega \tau_{M}}{\left(1-\omega^{2} \tau \tau_{M}+i \omega\left(\tau+\tau_{M}\left(1+K^{2} L_{D}^{2}\right)\right.\right.}
$$

Here $S$ is the electrode area, $\sigma_{0}$ is the average photoconductivity of the sample, $L_{D}$ is the diffusion length of photocarriers, $E_{D}=K k_{B} T / e$ is the diffusion field, $\tau_{M}=\varepsilon \varepsilon_{0} / \sigma_{0}$ is the Maxwell relaxation time, $\varepsilon$ is the dielectric constant, $\varepsilon_{0}$ is the free space permittivity, $\mathrm{k}_{\mathrm{B}}$ is the Boltzmann constant, and $\mathrm{e}$ is the elementary charge, $J_{n}(\Delta)$ is the Bessel function of the first kind of the $n$-th order.

First let us consider the case of the real gain factor

$\mathrm{H}=\mathrm{H}_{\mathrm{r}}$

$\operatorname{Im}\left(\mathrm{H}_{\mathrm{r}}\right)=0$

This situation is typical for conventional analog wideband amplifiers.

The phase conditions, i.e. phase shifts of the amplifier and feedback, define the operating frequency of generators in the oscillation theory. Namely, the sum of amplifier and feedback phase shifts must be multiple of $2 \pi$. We can always settle the phase of $\Delta$ to zero by the appropriate choice of the time origin. As follows from Eq. (7) the complex amplitude of the photocurrent is real value for modulation frequency equal to

$$
\omega_{g}=\omega_{m}=\left(\tau \tau_{M}\right)^{-1 / 2}
$$

It corresponds to the maximum in the frequency dependence of the photo-EMF amplitude.

The amplitude of oscillations in the conventional generators (electrical, optical, etc.) is usually defined by the amplifier's nonlinearity originating for large amplitudes. In our case the saturation of the non-steady-state photo-EMF for large $\Delta$ will confine the generation amplitude. As follows from Eqs. (5) and $(7)$ it is defined as:

$$
J_{0}(\Delta) J_{1}(\Delta) / \Delta=1 / \Xi
$$

Here we introduced the dimensionless gain factor $\Xi$ :

$$
\begin{aligned}
& \Xi=-\xi H_{r} E_{D} \\
& \xi=\frac{\pi S m^{2} \sigma_{0} R_{L} E_{D}}{U_{\pi}\left(1+K^{2} L_{D}{ }^{2}\right)}
\end{aligned}
$$

Equation (11) is nonlinear and can be solved graphically or numerically (Fig. 2). Oscillation with lowest amplitude occurs when dimensionless gain factor $\Xi$ is positive and reaches 2, i.e. $\Xi>2$ (Fig. 2). Increasing the gain factor from this value one can magnify the oscillation amplitude up to $\Delta \sim 2.4$. When gain factor reaches $\Xi \sim 60,180$, etc. oscillations with higher amplitudes ( $\Delta \sim 4.6,7.8$, etc.) become possible as well. As seen, each of these higher modes splits into two sub-modes with higher and lower amplitudes. Another feature is also seen from Fig. 2. The first mode (with lowest amplitude) is characterized by the soft regime of generation, i.e. such generation can appear as the result of very small deviation from the unstable equilibrium $\Delta=0$ (e.g. from the noise of load resistor). Higher modes are characterized by hard regime of generation and require sufficient initial deviation, which should be of order of the oscillation amplitude, i.e. 4.6, 7.8, etc. 

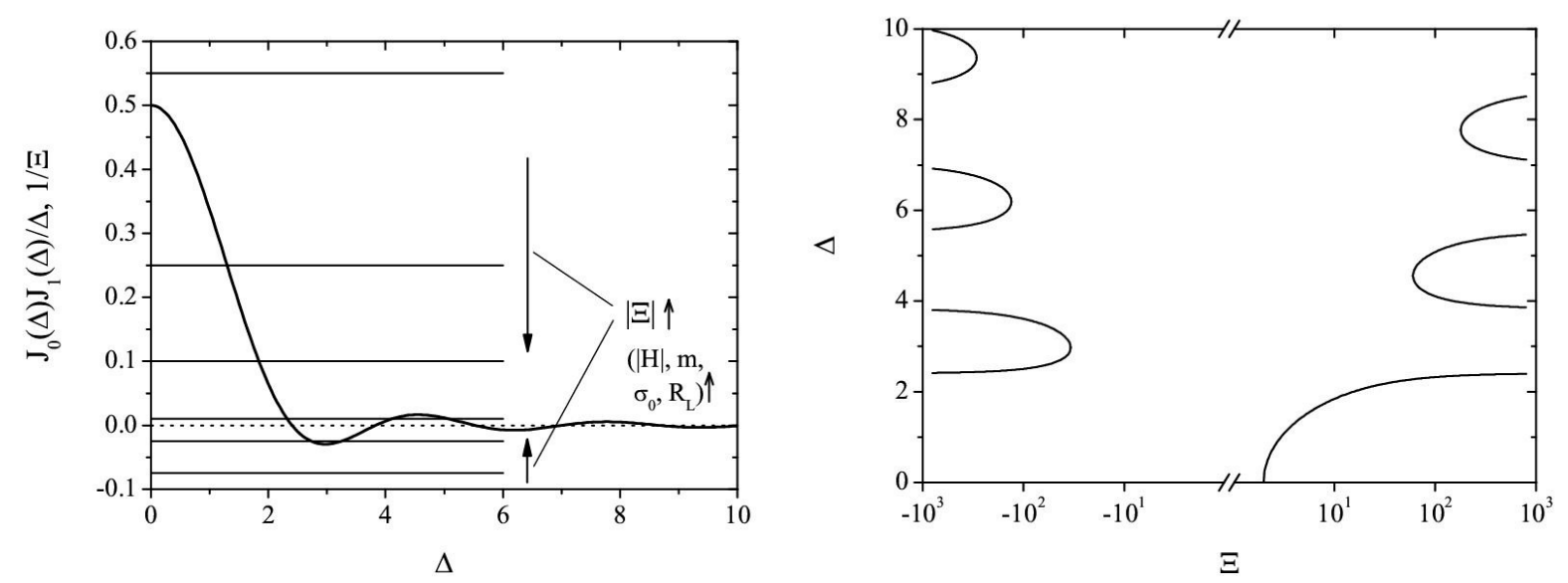

Figure 2. Graphic solution of Eq. (11) and corresponding dependence of the oscillation amplitude on the normalized gain factor.

The generation is also possible for negative gain factors $\Xi<0$ (Fig. 2 \}). In this case oscillations with lowest amplitude $\Delta \sim 3.0$ appear when gain factor reaches $\Xi \sim-34$. The behavior of modes is analogous to the one for positive gain factors. All the modes are characterized by hard regime of generation.

Now let us suppose that amplifier of the photo-EMF signal both increases the amplitude and introduces $\pi / 4$ phase shift:

$\mathrm{H}=\mathrm{H}_{\mathrm{r}} \exp (\mathrm{i} \pi / 4)$

Then the generation frequency equals

$\omega_{g}=\omega_{1}=\tau_{s c}^{-1}=\tau_{M}\left(1+K^{2} L_{D}^{2}\right)$

It should be noticed that this estimation is obtained for condition $\tau<<\tau_{\mathrm{M}}$ which is typical for high resistive semiconductors (e.g. for photorefractive $\mathrm{Bi}_{12} \mathrm{SiO}_{20}$ ). Frequency $\omega_{1}$ is the first cut-off frequency of the non-steady-state photo-EMF for the case of forced excitation of the signal [11] and it is the inverse value of the space charge formation time $\tau_{\mathrm{sc}}[19]$.

The amplitude of oscillation is calculated from Eq. (11) with

$\Xi=-\xi H_{r} E_{D} / \sqrt{2}$

Coefficient $\sqrt{2}$ appears here as the photo-EMF amplitude at frequency $\omega_{1}$ is $\sqrt{2}$ times lower than that at frequency $\omega_{\mathrm{m}}$. Since the amplitude of oscillations $\Delta$ is defined from the same equation as for the case of real $\mathrm{H}$ then its behavior with respect to gain factor $\Xi$ should be analogous to the one described above.

At last let the gain factor equal

$\mathrm{H}=\mathrm{H}_{\mathrm{r}} \exp (-\mathrm{i} \pi / 4)$

Then the oscillation frequency corresponds to the second cut-off frequency of the photo-EMF signal:

$$
\omega=\omega_{2}=\left(1+K^{2} L_{D}^{2}\right) / \tau
$$

and amplitude is deduced from Eqs. (11) and (16).

The experimental arrangement used for the investigation of the non-steady-state photo-EMF is shown in Fig. 3. The light from the conventional ND:YAG $\left(\lambda=532 \mathrm{~nm}, P_{\text {ou }} \approx 20 \mathrm{~mW}\right)$ laser was split into two beams forming the interference pattern with spatial frequency $\mathrm{K}$ and contrast $\mathrm{m}$ on the sample's surface. The signal beam was phase modulated with frequency $\omega$ and amplitude $\Delta$ by the electrooptic modulator ML-102A. The photocurrent arising in the sample generated the corresponding voltage on the load resistor $R_{L}=110 \mathrm{k} \Omega$. Then this voltage was amplified, measured by the analog-to-digital converter connected to computer, and applied back to the electrooptic modulator. The amplifier is characterized by the gain factor $40-80 \mathrm{~dB}$, phases 0 and $\pi$, flat frequency response in range $1.5 \mathrm{~Hz}-150 \mathrm{kHz}$, and highest output voltage of $100 \mathrm{~V}$. In our experiments we used conventional undoped $\mathrm{Bi}_{12} \mathrm{SiO}_{20}$ crystal. The sample has the following dimensions: $10 \times 4 \times 1 \mathrm{~mm}^{3}$. The silver paste electrodes $\left(3 \times 3 \mathrm{~mm}^{2}\right)$ are painted on the lateral surfaces. The interelectrode spacing equals $L=1 \mathrm{~mm}$. 


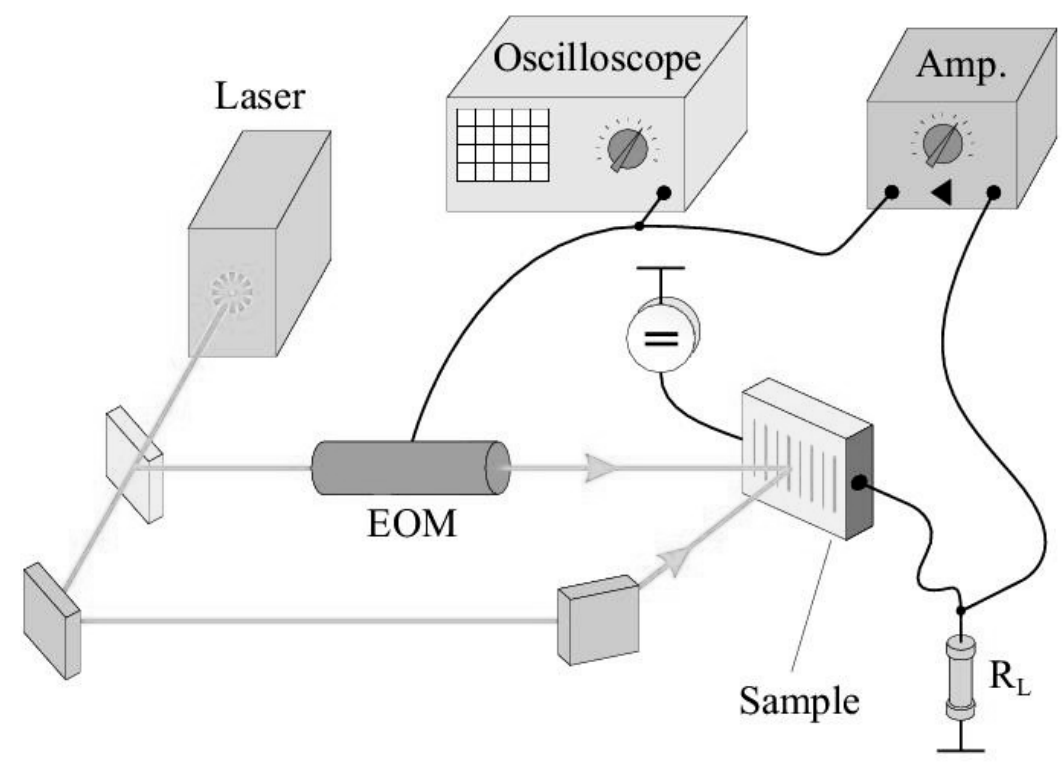

Figure 3. Simplified scheme of the experimental setup. EOM is the electrooptic modulator, Amp is the wideband amplifier with regulated gain factor.

First we have carried out standard measurements of the non-steady-state photo-EMF in $\mathrm{Bi}_{12} \mathrm{SiO}_{20}$ crystal. These experiments help us to find out necessary conditions for realization of the self excitation regime and analyze it. Frequency transfer function of the non-steady-state photo-EMF are presented in Fig. 4. The dependencies were measured for the zero external electric field (diffusion regime of signal excitation). We have approximated them by Eq. (7). The frequency of the maximum is one of the fitting parameters. The photocurrent is also characterized by the phase shift equal to $\pi$ (diffusion regime) at this frequency. It was found to be $\omega_{m} / 2 \pi=5.9 \mathrm{kHz}$ (for $E_{0}=0$ ). Namely this value is expected as the generation frequency in the experiments with positive feedback.

When feedback loop with appropriated phase shift and gain factor is created the oscillations of the current in crystal and oscillations of the optical phase of the signal beam arise. The minimum gain factors are the following: $H=-6.0 \times 10^{3}$ for the diffusion regime of the signal excitation. Let us calculate the corresponding theoretical values using the material parameters for $\mathrm{Bi}_{12} \mathrm{SiO}_{20}$ crystal: $s N_{D}=1.06 \times 10^{20} \mathrm{~J}^{-1} \mathrm{~m}$ $\mu /\left(\gamma N_{A}\right)=6.38 \times 10^{-11} \mathrm{~m}^{2} V^{-1}$. Assuming the illuminated electrode area to be equal to $S=3 \mathrm{~mm}^{2}$ (unexpanded beams, $\mathrm{E}_{0}=0$ ) we obtain the following estimations: $\mathrm{H}=-5.2 \times 10^{3}$ for the diffusion regime. One can note the good coincidence of the experimental and theoretical values.

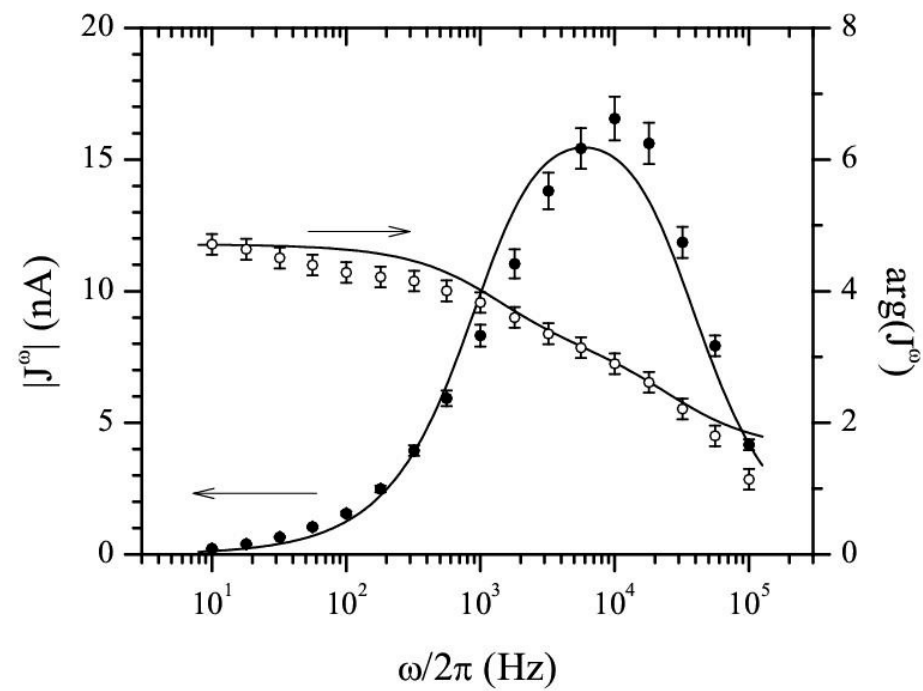

Figure 4. Amplitude and phase frequency transfer functions of the non-steady-state photo-EMF measured in $\mathrm{Bi}_{12} \mathrm{SiO}_{20}$ crystal $\left(\mathrm{E}_{0}=0, l_{0}=0.75 \mathrm{~W} / \mathrm{cm}^{2}, m=0.95, K=1.0 \mu \mathrm{m}^{-1}\right)$. 
To summarize, in this paper we have studied the new regime of the interferometer using detector based on the non-steady-state photo-EMF effect. Namely, oscillations of the optical phase are generated in the interferometer with the $\mathrm{Bi}_{12} \mathrm{SiO}_{20}$ detector and the optoelectronic feedback loop. The main parameters of such oscillations, i.e. amplitude and frequency, are defined mainly by the nonlinearity of the amplitude characteristic of the non-steady-state photo-EMF effect and photoelectric properties of the crystal and not by the experimental environment. This feature can be used for semiconductor testing applications.

Acknowledgements. M.B. and I.S. acknowledge the financial support from the Russian Science Support Foundation. This work was partially supported by the grant 07-02-01099 of the Russian Foundation for Basic Research.

\section{References}

1. R.L. Forward, Phys. Rev. D, 17, 379-390 (1978).

2. Stepanov, S. I., Reports on Progress in Physics, 57, 39-116 (1994).

3. S.I. Stepanov, I. A. Sokolov, Proc. of Second International Conference on Holographic Systems, Components and Applications, IEE, London, 311, 95-100 (1989).

4. M. Paul, B. Betz, W. Arnold, Appl. Phys. Lett. 50, 1569-1571 (1987).

5. M.P. Petrov, I.A. Sokolov, S.I. Stepanov, G.S. Trofimov, J. Appl. Phys. 68, 2216-2225, 1990.

6. M.I. Rabinovich and D.I. Trubetskov, Oscillations and waves in linear and nonlinear systems (Kluwer Academic Publishers, 1989).

7. D.G. Luenberger, Introduction to dynamic systems (John Wiley \& Sons, New York, 1979).

8. J. Bechhoefer, Rev. Mod. Phys. 77, 783 (2005).

9. S.A. Collins and K.C. Wasmundt, Opt. Eng. 19, 478 (1980).

10. S.I. Stepanov, I.A. Sokolov, G.S. Trofimov, V.I. Vlad, D. Popa, and I. Apostol, Opt. Lett. 15, 1239 (1990).

11. M.P. Petrov, I.A. Sokolov, S.I. Stepanov, and G.S. Trofimov, J. Appl. Phys. 68, 2216 (1990).

12. A.A. Kamshilin, J. Frejlich, and L. Cescato, Appl. Opt. 25, 2375 (1986).

13. A.A. Freschi and J. Frejlich, J. Opt. Soc. Am. B 11, 1837 (1994).

14. P.M. Garcia, K. Buse, D. Kip, and J. Frejlich, Opt. Commun. 117, 235 (1995).

15. V.P. Kamenov, K.H. Ringhofer, B.I. Sturman, and J. Frejlich, Phys. Rev. A 56, R2541 (1997).

16. E.V. Podivilov, B.I. Sturman, S.G. Odoulov, S. Pavlyuk, K.V. Shcherbin, V.Ya. Gayvoronsky, K.H. Ringhofer, and V.P. Kamenov, Phys. Rev. A 63, 053805 (2001).

17. P.M. Garcia, A.A. Freschi, J. Frejlich, and E. Kraetzig, Appl. Phys. B 63, 207 (1996).

18. M. Gorkunov, B. Sturman, M. Luennemann, and K. Buse, Appl. Phys. B 77, 43 (2003).

19. M.P. Petrov, S.I. Stepanov, and A.V. Khomenko, Photorefractive crystals in coherent optical systems (Springer-Verlag, Berlin, 1991). 